\title{
Vascular reconstruction and long-term outcome of living domino liver transplantation in children
}

\author{
Seiichi Shimizu', Seisuke Sakamoto ${ }^{1}$, Yasuyuki Kameoka ${ }^{1}$, Kotaro Mimori ${ }^{1}$, Hajime Uchida ${ }^{1}$, Yusuke Yanagi $^{1}$, \\ Akinari Fukuda ${ }^{1}$, Reiko Horikawa ${ }^{2}$, Mureo Kasahara ${ }^{1}$ \\ ${ }^{1}$ Department of Organ Transplantation Center, National Center for Child Health and Development, Tokyo, Japan \\ ${ }^{2}$ Department of Endocrinology and Metabolism, National Center for Child Health and Development, Tokyo, Japan
}

Background: A native liver of maple syrup urine disease (MSUD) patients can be used as a graft to non-MSUD patients with endstage liver disease because an extrahepatic enzyme activity can sufficiently maintain metabolic functions of these patients, socalled the second recipient. Operational procedures of second recipient in domino liver transplantation (DLT) and long-term outcome of these patients can impact on making a decision to offer this procedure to the others.

Methods: Six second recipients of DLT (patients' age, 42.5 months old at DLT; range, 22-169 months old) received a graft of the native liver from MSUD patients at National Center for Child Health and Development between June 2014 and April 2020. We reviewed the operational procedures including vascular reconstructions and outcomes of second recipients of DLT (follow-up, 5.5 years; range, 0.4-6.3 years).

Results: Five of the six second recipients had a whole liver and one had a right lobe graft. The median operative time was 457 minutes (range, 303-750 minutes) and cold ischemia time was 264 minutes (range, 250-350 minutes). On the back table, multiple hepatic veins of the graft were unified into single orifice without any vein grafts in all cases. The recipient's hepatic vein orifice was anastomosed to a newly-created orifice of the graft. For portal vein reconstruction, one case needed an autologous left external iliac vein as an interpositional vein graft. Arterial reconstruction was performed by the anastomosis between donor's and recipient's proper hepatic artery. The median hospital stay was 31 days (range, 19-80 days) without any primary non function and vascular or bile duct complications. Two patients had acute cellular rejection. All recipients were doing well without the elevation of valine, leucine, or isoleucine in the amino acid analysis.

Conclusions: Metabolic functions of second recipients have maintained within normal ranges under unrestricted protein diet. MSUD liver can be safely used and it may expand a donor-pool as an alternative graft in pediatric liver transplantation.

Corresponding author: Seiichi Shimizu

E-mail: shimizu-se@ncchd.go.jp

() The Korean Society for Transplantation

This is an Open Access article distributed under the terms of the Creative Commons Attribution Non-Commercial License (http://creativecommons.org/licenses/by-nc/4.0/) which permits unrestricted non-commercial use, distribution, and reproduction in any medium, provided the original work is properly cited. 